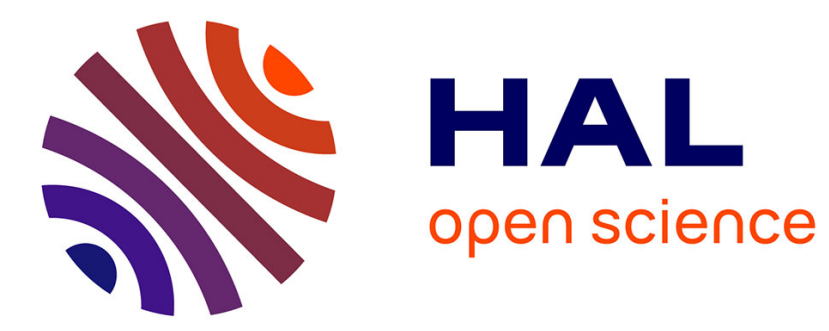

\title{
Collisional blockade in microscopic optical dipole traps
}

Nicolas Schlosser, Georges Reymond, Philippe Grangier

\section{To cite this version:}

Nicolas Schlosser, Georges Reymond, Philippe Grangier. Collisional blockade in microscopic optical dipole traps. Physical Review Letters, 2002, 89 (2), pp.023005. 10.1103/PhysRevLett.89.023005 . hal-00554211

\section{HAL Id: hal-00554211 \\ https://hal-iogs.archives-ouvertes.fr/hal-00554211}

Submitted on 10 Jan 2011

HAL is a multi-disciplinary open access archive for the deposit and dissemination of scientific research documents, whether they are published or not. The documents may come from teaching and research institutions in France or abroad, or from public or private research centers.
L'archive ouverte pluridisciplinaire HAL, est destinée au dépôt et à la diffusion de documents scientifiques de niveau recherche, publiés ou non, émanant des établissements d'enseignement et de recherche français ou étrangers, des laboratoires publics ou privés. 


\title{
Collisional Blockade in Microscopic Optical Dipole Traps
}

\author{
N. Schlosser, G. Reymond, and P. Grangier* \\ Laboratoire Charles Fabry de l'Institut d'Optique, UMR 8501 du CNRS, 91403 Orsay, France
}

(Received 4 February 2002; published 19 June 2002)

\begin{abstract}
We analyze the operating regimes of a very small optical dipole trap, loaded from a magneto-optical trap, as a function of the atom loading rate, i.e., the number of atoms per second entering the dipole trap. We show that, when the dipole trap volume is small enough, a "collisional blockade" mechanism locks the average number of trapped atoms on the value 0.5 over a large range of loading rates. We also discuss the "weak loading" and "strong loading" regimes outside the blockade range, and we demonstrate experimentally the existence of these three regimes.
\end{abstract}

DOI: 10.1103/PhysRevLett.89.023005

PACS numbers: $32.80 . P \mathrm{j}, 03.67 . \mathrm{Lx}, 42.50 . \mathrm{Vk}$

There is currently a strong interest for the manipulation of individual neutral atoms in microscopic optical dipole traps [1-5]. Besides their fundamental interest, the manipulations of individual quantum objects may open the way to controlled engineering of the quantum state of small sets of trapped particles, in order to encode and process information at the quantum level [6-9]. We have demonstrated recently that it is possible to load and detect individual atoms in an optical dipole trap with a submicrometer size [1]. The dipole trap is initially loaded from a very low density magneto-optical trap (MOT), which cools the atoms and allows us to detect them easily from the induced fluorescence. Because of the extremely small trapping volume, only one atom can be loaded at a time, resulting in strongly sub-Poissonnian statistics of the number $N$ of atoms in the trap. This corresponds to having either zero or one atom in the trap, with equal probabilities. However, these results [1] left two crucial questions unanswered.

(i) What is the physical mechanism responsible for the observed self-limitation of the number of trapped atoms? More specifically, does this mechanism involve new types of inelastic collisions, or does it correspond to known mechanisms observed in a new range of parameters?

(ii) Is the self-limitation a universal effect, or it is possible to "bypass" it for very large loading rates of the dipole trap? In that case, the self-limitation would appear as a "collisional blockade" mechanism, that occurs in a specific range of trap parameters.

In this Letter, we provide answers to these two questions. First, we show that there is indeed a "blockade" effect, which is due to an original combination of the well-studied behavior of dipole traps in the presence of MOT light [10], and of new features that are specifically due to the very small volume of our trap. In particular, we will show that the novel blockade regime appears only if the trap is small enough. Then we present a simple theoretical analysis, which allows us to identify three operating regimes ("weak loading," blockade, and "strong loading"), as a function of the number of atoms that enter the dipole trap area. Finally, we will present experimental evidence for the observation of these three regimes in our dipole trap, which is characterized by a beam waist $w_{0} \sim 0.7 \mu \mathrm{m}$. We conclude that in the strong loading regime it is possible to have extremely small and dense nanoclouds containing a few tens of atoms.

We consider an optical dipole trap containing $N$ trapped atoms. The number $N$ will vary as a function of the loading rate $R$, the one-body decay $-\gamma N$ (essentially due to collisions with fast atoms from the background gas), and the two-body decay $-\beta^{\prime} N(N-1)$ (that is due to a mixture of various inelastic collisional mechanisms, analyzed in detail in [10]). The equation for $N$ is thus:

$$
\frac{d N}{d t}=R-\gamma N-\beta^{\prime} N(N-1) .
$$

Since this equation is written for the atom number and not for the atomic density, the value of $\beta^{\prime}$ is inversely proportional to the volume of the trap [10]: the smallest the trap, the largest $\beta^{\prime}$. In the steady state, one can immediately identify two regimes. (i) Weak loading: For small values of $R$, the effect of the collisional term is negligible, so that $\langle N\rangle \sim R / \gamma$. (ii) Strong loading: For large values of $R,\langle N\rangle$ becomes large, and the collisional term becomes important. The average number of atoms is then limited by the collisions, so that $\langle N\rangle \sim \sqrt{R / \beta^{\prime}}$.

The crossover between weak and strong loading is defined by a critical atom number $N_{c}=\gamma / \beta^{\prime}$, associated with a critical loading rate $R_{c}=\gamma^{2} / \beta^{\prime}$ above which the collisional term becomes dominant (see Fig. 1). As long as $N_{c} \gg 1$, collisions play a role only when $\langle N\rangle$ is large, and one has simply a reduction in the slope of $\langle N\rangle$ vs $R$. But when $N_{c} \ll 1$, this reduction would appear even if $\langle N\rangle \ll 1$, which is not physically acceptable because at least two atoms are needed for a collision. In this case, collisions play a role as soon as there are two atoms in the dipole trap: This is the collisional blockade regime.

The simplest way to analyze this regime is to use a classical Monte Carlo simulation, with random atom arrivals, including one-body and two-body decays. In the simulation, one has to choose whether a collision ejects both atoms from the trap, or only one; good agreement with the experiment is obtained by assuming that in the collision both atoms are ejected from the trap. This is also consistent with what is expected from the underlying collisional 


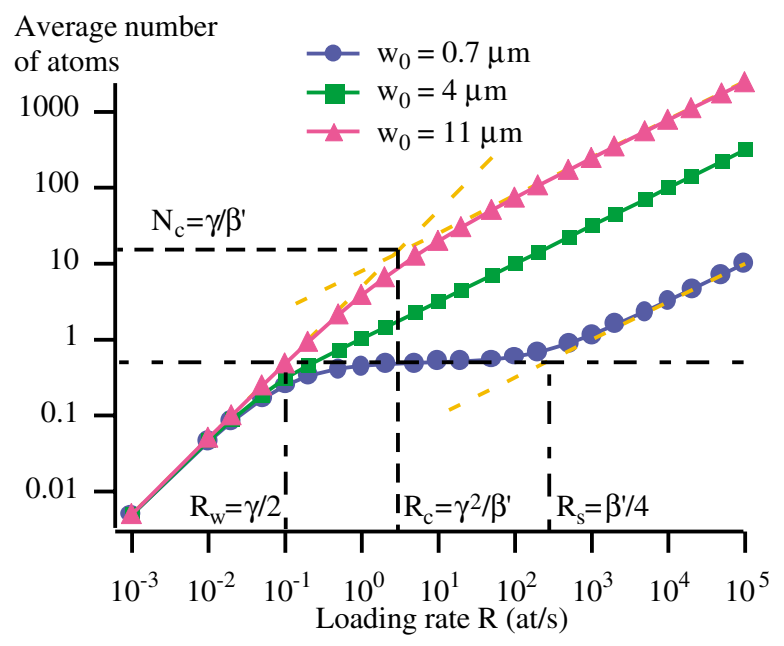

FIG. 1 (color online). Average number of atoms in the trap as a function of the loading rate $R$ for different values of the waist. $w_{0}=11 \mu \mathrm{m}$ corresponds to a standard configuration. For $w_{0}=0.7 \mu \mathrm{m}$, the blockade regime is clearly seen.

mechanisms [11], even if one-atom losses have also been observed in other geometries [12]. Consequently, as long as one stays in the blockade regime, the number of atoms is either zero or one, and the arrival of an atom in the trapping volume is responsible for either a loading or a loss event. It is then clear that the average number of atoms should be $\langle N\rangle=0.5$, which is indeed the observed value [1]. We note that in the presence of one-body losses, we would reach an average number of atoms $0.5<\langle N\rangle<1$, contrary to the experiment. When $\langle N\rangle=0.5$ the loading rate limits are given by $R_{w}=\gamma / 2$ on the weak loading side, and $R_{s}=\beta^{\prime} / 4$ on the strong loading side (see Fig. 1). The blockade regime appears when $R_{w}<R_{s}$, i.e., $N_{c}<0.5$, as a "plateau" where the average atom number is fixed. We will show now that this plateau can extend over several orders of magnitude in the loading rate, provided that $\beta^{\prime}$ is large enough.

In order to get quantitative predictions, one has to introduce numerical values for $\gamma$ and $\beta^{\prime}$. We will use $\gamma=0.2 \mathrm{~s}^{-1}$, which is consistent with our observations, and with the background pressure in our vacuum chamber (a few $10^{-9}$ Torr). The value of $\beta^{\prime}$ will be taken from Ref. [10], which leads to $\beta^{\prime}=1000 \mathrm{~s}^{-1}$ for our trapping volume. This value is compatible with our observations. We note that $\beta^{\prime}$ reduces very quickly with the trap size (the volume of the trap is proportional to $w_{0}^{4}$ ); e.g., it drops to $\beta^{\prime}=0.016 \mathrm{~s}^{-1}$ for a more "standard" beam waist $w_{0}=11 \mu \mathrm{m}$. Figure 1 shows in log-log scale the number of trapped atoms as a function of the loading rate. This figure shows clearly that the blockade effect appears for a trap size that is typically smaller than $4 \mu \mathrm{m}$. In our operating conditions, it extends over about 3 orders of magnitude in loading rates, typically between $R=0.1$ and 100 atoms $/ \mathrm{s}$. Figure 2 shows the histograms of the number of trapped atoms obtained from the Monte Carlo
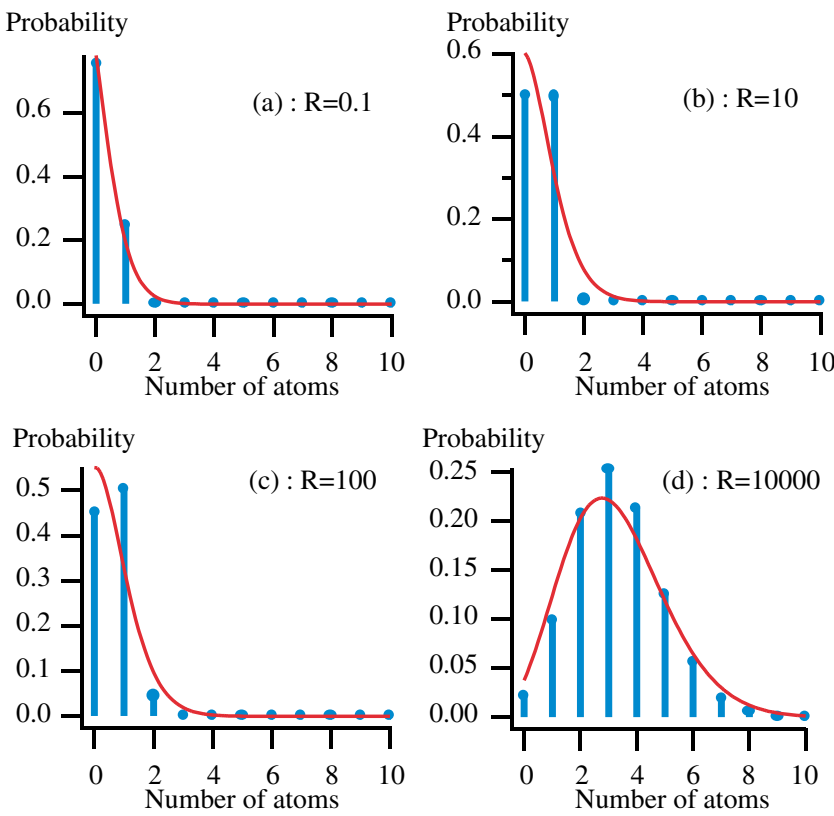

FIG. 2 (color online). Histograms of the number of trapped atoms for different values of the loading rate $R$, calculated with the Monte Carlo simulation. The continuous curves are the Poisson law for the same average value.

simulations. All distributions are actually sub-Poissonian, but this effect is particularly clear in the blockade regime, where only $N=0$ and $N=1$ have a significant probability, which is close to $1 / 2$. The variance is then 0.25 , i.e., $0.5\langle N\rangle$ or "3 $\mathrm{dB}$ squeezing" [1].

It is worth noticing that the characteristic lifetime of the trapped atoms is decreasing proportionally to the loading rate from one histogram to the other. This means that the observation time needed to see a constant number of atoms in the trap is shorter and shorter, and it drops below $1 \mathrm{~ms}$ roughly at the turning point between the blockade and the strong loading regimes. This creates a significant experimental problem, because the relative shot-noise associated with the number of photons detected during this time becomes larger and larger. How this problem was solved will be discussed below.

In order to check these results experimentally, we used the setup of Fig. 3 (see also [1]), which consists of a strongly focused dipole trap loaded from a magnetooptical trap. The MOT is loaded from an atomic beam, slowed down by chirped cooling using the usual "slower" and "repumper" laser diodes. In the following, the MOT density, which is proportional to the loading rate of the dipole trap, will be changed over many orders of magnitude. The highest values (about $10^{10} \mathrm{~cm}^{-3}$ ) are obtained with standard parameters, while the lowest ones (about $10^{5} \mathrm{~cm}^{-3}$ ) are obtained by turning off the slowing beams and the MOT magnetic field, and decreasing the oven temperature.

The focusing objective consists of two parts, which first focus the light inside the MOT and then recollimates the 


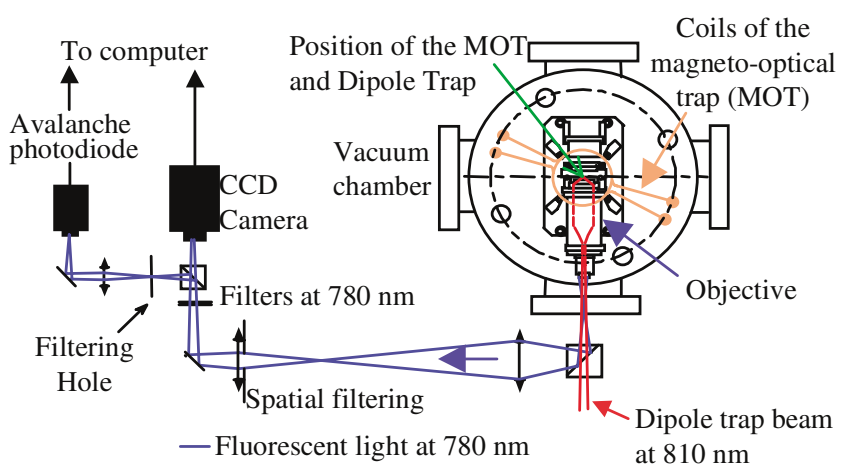

FIG. 3 (color online). Main features of the experimental setup. For simplicity, the slowing and MOT beams are not represented. The dipole trap beam is generated by a titanium:sapphire laser.

beam for diagnostic purposes. The numerical aperture of 0.7 gives a diffraction-limited resolution of $0.7 \mu \mathrm{m}$ for the focusing part. The light beam injected in the objective comes from a cw, frequency stabilized titanium-sapphire laser, and it is brought to the setup by using an optical fiber. A wide range of power and wavelength is thus available, and the dipole trap is usually operated in the "FORT" (far off resonance) regime around $810 \mathrm{~nm}$ [13], with a few $\mathrm{mW}$ input power.

The fluorescence from the trapped atoms excited by the MOT light is collected by the same objective focusing down the beam (see Fig. 3), and gives a magnified image of the trap on a CCD camera. Since the CCD camera has a slow response time, a photon-counting avalanche photodiode (APD) is used in parallel, and monitors only the light coming from the trap region: typically, the onemicron diameter dipole trap is imaged onto a fifty-micron pinhole. We note that all the photon counting is done at the MOT wavelength at $780 \mathrm{~nm}$ : The stray light elimination is obtained only by spatial filtering. On the other hand, the residual dipole trap stray light at $810 \mathrm{~nm}$ is eliminated by an interference filter. An atom in the dipole trap sees a large light shift, and therefore its fluorescence rate is weaker than the same atom in the MOT. This can easily be seen by suddenly turning off the dipole trap: Before the atom leaves the observation area (a few cubic microns in size), its fluorescence rate shows a sharp increase that appears as a flash of light on the APD. The typical detection rate from a single atom in the dipole trap is about 5000 counts per second (cps), while it is about $20000 \mathrm{cps}$ in the MOT alone.

In the weak loading regime, the fluorescence photons can be counted in $10 \mathrm{~ms}$ time bins, and the duration of the plateaus (typically a few seconds [1]) is essentially limited by the one-body decay introduced above. When the loading rate is increased, the probability to have one atom increases also, and then locks on the value 0.5. Simultaneously, the duration of the plateaus becomes shorter and shorter. The corresponding histogram is shown in Fig. 4(a). In Fig. 5, we show the probability to have one

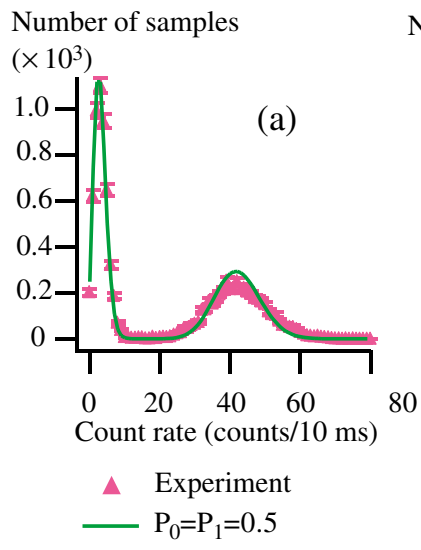

Number of samples

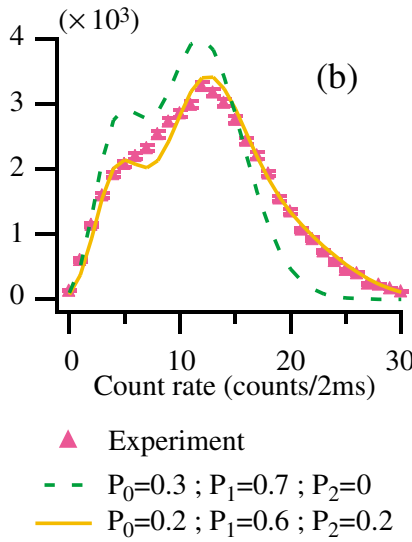

FIG. 4 (color online). Histograms in the collisional blockade regime (a) and in the intermediate regime (b). Fits of these results give the probability $P_{n}$ of trapping $n$ atoms. The dash curve obtained with $P_{2}=0$ is unable to fit the experimental points in the intermediate regime.

atom (deduced from the histogram) and the duration of the plateaus (obtained by computing the autocorrelation of the counting signals), as a function of the loading rate, which is controlled by changing the MOT magnetic field. The blockade regime is clearly apparent on these curves.

If the loading rate is increased further, the number of atoms starts fluctuating faster than the $10 \mathrm{~ms}$ counting time. One has thus to reduce this time, but the photon noise becomes a serious issue: Only typically five photons are detected in $1 \mathrm{~ms}$, and it becomes difficult to separate the zero and one-atom peaks. Nevertheless, Fig. 4(b) shows a histogram taken with a time bin of $2 \mathrm{~ms}$, in the intermediate regime that is close to the upper limit of the blockade regime. Fitting the histogram reveals that a two-atom contribution is appearing, though it is still not very large.

Finally, one may go to high loading rates, and decrease the time bin to $0.5 \mathrm{~ms}$. The steps due to individual atoms are no more resolved, but the number of atoms can be

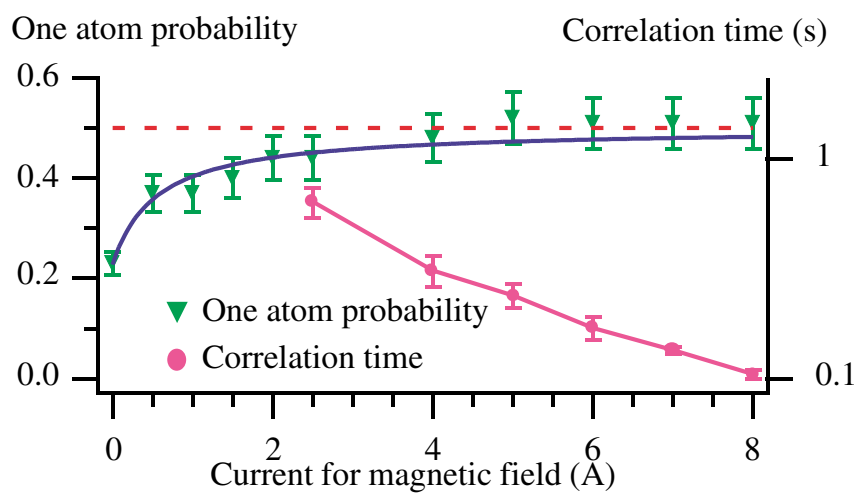

FIG. 5 (color online). Evolution of the autocorrelation time and one-atom probability showing the blockade regime. For small currents, the autocorrelation time is not easily measurable because a long integration time is necessary. 
determined by studying the statistical distribution of photons coming from the trapped atoms. A first part due to the background light is a Poisson law, and the probability of counting $n_{b}$ photons from the background light is $P_{b}\left(n_{b}\right)=\Pi\left(n_{b}, N_{b}\right)$, where $\Pi\left(n, N_{b}\right)$ is a Poisson law for an average value of the background equal to $N_{b}$.

In order to calculate the probability of counting $n_{s}$ photons coming from the trap, we make the assumption of a Poisson law for the number of atoms around $N_{\text {at }}$, and for the number of photons per atoms in the sampling window around $N_{\mathrm{ph}}$. Actually, neither the atom nor the photon distributions are exactly Poissonian, but this turns out to be a good enough approximation for our purpose [see Fig. 2(d) for the atomic distribution]. When the number of trapped atoms is $n_{\text {at }}$ with the probability $p_{\text {at }}\left(n_{\text {at }}\right)=\Pi\left(n_{\text {at }}, N_{\text {at }}\right)$, the probability of getting $n$ photons is $\left.P_{s}(n)\right|_{n_{\mathrm{at}}}=\Pi\left(n, N_{\mathrm{ph}} n_{\mathrm{at}}\right)$, and the probability of measuring $n_{s}$ photons coming from the trap becomes: $P_{s}\left(n_{s}\right)=\sum_{n_{\mathrm{at}}=0}^{+\infty} \Pi\left(n_{\mathrm{at}}, N_{\mathrm{at}}\right) \times \Pi\left(n_{s}, N_{\mathrm{ph}} n_{\mathrm{at}}\right)$ The probability of detecting $n=n_{s}+n_{b}$ photons coming either from the trap or from the background is thus:

$$
P(n)=\sum_{k=0}^{n} P_{s}(k) P_{b}(n-k) .
$$

The experimental measurement of the two distributions (light from background or trapped atoms) gives the average value of the background $N_{b}$ and of the light coming from trapped atoms $N_{\mathrm{ph}} N_{\text {at }}$ (see Fig. 6). The compound Poisson distribution $P(n)$ given by (2), depending on $N_{b}, N_{\text {at }}$ and $N_{\text {ph }}$, can be fitted on the data with only one free parameter. We get finally the average number of atoms in the trap $N_{\text {at }}$ and the average number of photons per atom $N_{\mathrm{ph}}$, compatible with the one we directly measured in the weak loading regime. The number of atoms depends on the loading rate, and takes typical values between 1 and 10 in the upper $(F=2)$ hyperfine level of the ground state. We note that, by lowering the MOT repumper, the atoms can be transferred in the lower $(F=1)$ level, where up to 40 atoms have been observed experimentally.

It is thus clear that the experiment recovers all the operating domains (weak loading, blockade, strong loading) that were predicted by the simple model discussed above. Though very different, these domains result essentially from the same physics, and represent simply the various ranges where the three terms in Eq. (1) successively become dominant.

As a conclusion, we have analyzed theoretically and experimentally a collisional blockade mechanism that locks the atom number or either zero or one in a very small dipole trap. This effect can be analyzed using the standard collisional mechanisms already known in combined MOT and

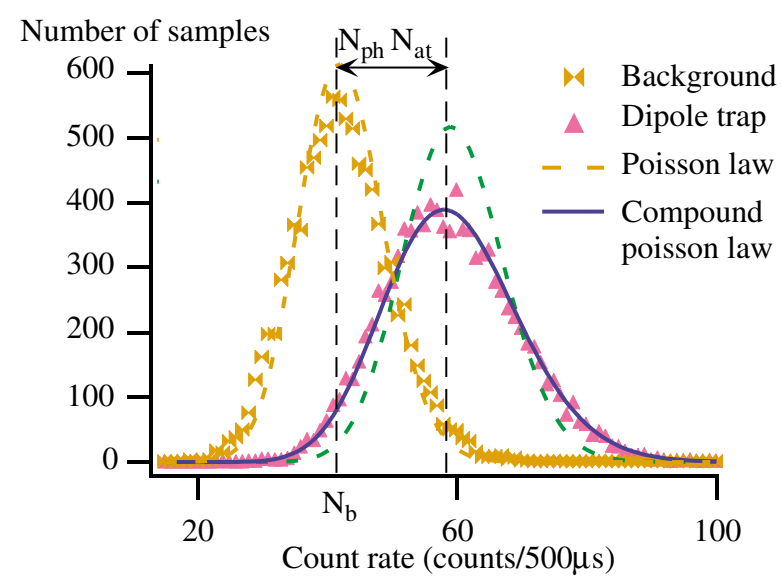

FIG. 6 (online color). Photon statistics for an average number of nine atoms. The background light is correctly fitted with a Poisson law but the light coming from the trap is a compound Poisson law.

dipole traps. It results actually from the fact that the trap volume is so small that collisions become the dominant loss mechanism as soon as there are two atoms in the trap. We have also shown that it is nevertheless possible to bypass the blockade regime, opening the possibility to have a large number of cold atoms in an extremely confined volume.

We thank Pierre Pillet and Jacques Vigué for useful discussions. This work was carried out in the framework of the European projects "QUBITS" (IST/FET/QIPC) and "QUEST" (IHP/RTN).

*Email address: Philippe.Grangier@iota.u-psud.fr Web page: http://www.iota.u-psud.fr/ grangier

[1] N. Schlosser, G. Reymond, I. Protsenko, and P. Grangier, Nature (London) 411, 1024 (2001).

[2] S. Kuhr et al., Science 293, 278 (2001).

[3] M. Greiner et al., Nature (London) 415, 39 (2002).

[4] A. C. Doherty, T. W. Lynn, C. J. Hood, and H. J. Kimble, Phys. Rev. A 63, 013401 (2001).

[5] P. W. H. Pinkse, T. Fischer, P. Maunz, and G. Rempe, Nature (London) 404, 365 (2000).

[6] C. A. Sackett et al., Nature (London) 404, 256 (2000).

[7] A. Rauschenbeutel et al., Phys. Rev. Lett. 83, 5166 (1999).

[8] T. Calarco et al., J. Mod. Opt. 47, 2137 (2000).

[9] G. K. Brennen, I. H. Deutsch, and P. S. Jessen, Phys. Rev. A 61, 062309 (2000).

[10] S. J. M. Kuppens et al., Phys. Rev. A 62, 013416 (2000).

[11] Jacques Vigué (private communication).

[12] B. Ueberholz et al., J. Phys. B 33, L135-L142 (2000).

[13] J. D. Miller, R. A. Cline, and D. J. Heinzen, Phys. Rev. A 47, R4567 (1993). 